\title{
Comparison of Multiple Spacecraft Configuration Designs for Coordinated Flight Missions
}

\author{
Federico Fumenti and Stephan Theil
}

\begin{abstract}
Coordinated flight allows the replacement of a single monolithic spacecraft with multiple smaller ones, based on the idea of distributed systems. According to the mission objectives and in order to ensure a safe relative motion, constraints on the relative distances need to be satisfied. At first a proper orbit design can limit the differential perturbations, then through corrective maneuvers their induced differential drifts can be canceled. In this work several designs are surveyed, defining the initial configuration of a group of spacecraft while counteracting the differential perturbations. For each of the investigated designs the focus is on the number of deployable spacecraft and on the possibility to ensure safe relative motion through station keeping of the initial configuration, with particular attention to the required $\Delta V$ budget and the constraints violations.
\end{abstract}

\section{Introduction}

In recent years the interest in spacecraft coordinated flight increased more and more due to the numerous potential advantages associated with the replacement of a single monolithic object with several smaller ones. A group of small spacecraft working together could enhance scientific observations, augment flexibility and redundancy, reduce costs and risks and overcome physical limitations. At the same time though, new challenges are introduced concerning for example the sharing of data, the communication and the relative motion among the objects. Focusing on this last aspect, it is trivial that when multiple objects are considered, much attention must be paid on the way they move w.r.t. each other. Constraints might be applied to ensure a safe relative motion and according to their strictness the two branches of formation flight and cluster flight can be distinguished. Due to many technical limitations the spread of the two architectures did not evolve in the same way, up to the point that

Federico Fumenti, Stephan Theil

DLR, Institute of Space Systems, Robert Hooke Str. 7, 28359, Bremen

e-mail: federico.fumentiedlr.de 
formations are being largely studied and successfully implemented, while clusters are nowadays still under investigation.

In a formation of satellites the relative configuration is fixed and control actions are required to maintain it. In a typical mission scenario several sensors and instruments demand for tight relative positions to cooperate, but cannot be allocated on the same spacecraft because the required relative distances are too wide and the resulting spacecraft exceeds the launch vehicle capabilities. To cope with this issue the devices can be distributed on different spacecraft which fly together while guaranteeing the satisfaction of the relative distances requirements through the use of control actions. Examples of missions implementing the formation flight concept can be found in TanDEM-X [20], PRISMA [21] and GRACE [26].

In the case of a cluster instead there is no need for precise geometry, because the successful outcome of the mission does not strictly depend on specific relative configuration as in the formation flight case. As long as the distances among the spacecraft are held within a maximum and a minimum value to ensure inter-modules communication and avoid collisions, respectively, no control action is required. It all results in less strict relative motion requirements, with a consequent relaxed intervention from the control system. In a typical scenario each member of the cluster allocates a different functionality, like communications, data storage, power generation, etc. and all the functionalities are shared through wireless connections. Examples for potential application of the cluster flight can be identified in the missions PLEIADES [15] and SAMSON [13].

In the design of a coordinated spacecraft based-mission an extremely important task is the definition of the initial configuration, since the application of proper constraints on the relative states will impress a particular desired behaviour to the evolution of the relative motion. Over time corrective maneuvers are required to counteract the changes in the initial relative geometry deriving from the differential perturbations. Therefore, to limit the required fuel and the missions costs, it is highly desirable to have orbits that naturally satisfy the relative motion constraints. Over the years many authors worked on the development and improvement of mathematical models to easily grasp the evolution of the relative motion and simplify the application of the required constraints (see e.g., [14, 6, 27, 5, 25, 29, 17, 16]).

Once the initial configuration is defined and the spacecraft are deployed, a station keeping approach could be used to cancel the drifts induced by the differential perturbations. Indeed the initial states ensure satisfaction of the distance constraints and can be seen as reference states to be tracked. This approach is certainly meaningful in a formation, where the relative geometry constraints limit considerably the tolerable differential drifts. Less clear is instead the benefit of cluster keeping through station keeping. In this case the loose constraints involve that the distance boundaries are infrequently violated and when this happens the drift from the reference state could be so large that the recomputation of a new reference state could become more meaningful than the station keeping of the old one. The goal of this work is to evaluate if and how much the station keeping logic could be beneficial for coordinated flight missions characterized by a different number of spacecraft and different distance boundaries. To study and implement the station keeping logic, it can be 
advantageous expressing the relative motion through relative orbital elements, since the orbital elements have a slow variation over time and corrections of specific elements with theoretically no effect on the others can be obtained using impulsive control (see e.g., [25, 24, 23, 18, 3, 22, 12]).

According to the type of constraints initially imposed, various initial configurations can be found, differing from each other for the number of deployable spacecraft, their relative geometry and the effort, in terms of $\Delta V$, they require for station keeping. A survey and comparison of such initial configurations is the topic of this work, which is organized as follows. Section 2 introduces the problem and the techniques used to define the initial states of a group of spacecraft. Section 3 describes how the comparison has been set and the key parameters used. The results of the study are then presented in Section 4, while Section 5 reports the final conclusions.

\section{Problem Statement}

The successful outcome of a coordinated flight-based mission strictly depends on the relative motion among the involved spacecraft and to ensure satisfaction of relative motion constraints, much attention must be put on the control actions and on the counteraction of the differential perturbations. To reduce the fuel expenditure specific constraints can be imposed in the definition of the initial conditions, according to the desired behavior required by the mission goals.

From the literature research it emerged that several techniques to define the relative initial conditions of a group of satellites are available, hence it has been decided to examine and compare them to see if and how the computed initial conditions could be used for the deployment of a cluster of objects.

The techniques for the initial conditions (TIC) that have been studied are introduced in the upcoming sections but at first, in order to make their comparison meaningful, a common test setup is identified: it is supposed that the cluster is centered in a virtual point (VP) and it is assumed that minimum distance constraints (MinDC) as well as maximum distance constraints (MaxDC) are in place. In particular, to prevent collisions a minimum distance $D_{\min }$ must be guaranteed between any pair of spacecraft, while escaping drifts are avoided through upper bounding the distance of a spacecraft from the VP by a maximum value $D_{\max }$. Denoting with $d_{s}$ and $d_{r}$ the generic distances between any two spacecraft of the cluster and between a spacecraft and the VP, respectively, the MinDC and the MaxDC are given by:

$$
\begin{aligned}
& d_{s}>D_{\min } \\
& d_{r}<D_{\max } .
\end{aligned}
$$

The VP is supposed to move on a low Earth orbit (LEO) and its initial state is defined in Table 1 in terms of osculating keplerian elements $\mathbf{\propto}_{V P}^{K}\left(t_{0}\right)=(\text { a e i } \omega \Omega M)^{T}$.

The description of each technique is structured in two parts. At first the basic logic is introduced while using the simple chief-deputy framework. The chief is the VP and the state of the deputy is defined such that their relative distance is bounded 
Table 1 Initial state of the virtual point.

\begin{tabular}{crc}
\hline Element & Value & Units \\
\hline semimajor axis $-a$ & 7000.92 & $\mathrm{~km}$ \\
eccentricity $-e$ & 0.01 & \\
inclination $-i$ & 50.99 & $\mathrm{deg}$ \\
right ascension of the ascending node $-\Omega$ & 11.48 & $\mathrm{deg}$ \\
argument of perigee $-\omega$ & 19.12 & $\mathrm{deg}$ \\
mean anomaly $-M$ & 21.00 & $\mathrm{deg}$ \\
\hline
\end{tabular}

by $D_{\min }$ and $D_{\max }$. Afterwards, it is shown how that same logic can be adapted to configure a cluster of $n_{m}$ modules while also maximizing $n_{m}$. As a matter of fact for the case of a cluster the simple chief-deputy approach does not fit very well as it is, and for several reasons. In the first place, from the stated assumptions it is clear that there is no need to lower bound the distances $d_{r}$ and to upper bound the distances $d_{s}$. Secondly, when several modules are placed into the cluster and the chief-deputy technique is applied to every module, information about the motion of the spacecraft w.r.t. the VP are available, but nothing can be said about a deputy-deputy type of motion. Plus, the idea of studying the motion of each agent with respect to all the others is inconceivable, since as $n_{m}$ grows the problem quickly becomes extremely complex and unmanageable.

In order to lighten up the descriptions of the investigated techniques in the upcoming sections, it wants to be revised here the distinction between keplerian and non-singular orbital elements, since both of them will be used. The keplerian set of elements $\mathbf{e}^{K}$ has been actually already introduced through Table 1 , while the nonsingular set is given by $\mathbf{a}^{N}=\left(a u e_{x} e_{y} i \Omega\right)^{T}$, where $e_{x}=e \cos \omega$ and $e_{y}=e \sin \omega$ are the components of the eccentricity vector $\mathbf{E}=\left(e_{x} e_{y}\right)^{T}$ and $u=\omega+M$ is the mean argument of latitude. In addition, it is also worth recalling that when two spacecraft are considered and their states are expressed in terms of orbital elements, the relative motion of the deputy $\mathrm{D}$ w.r.t. the chief $\mathrm{C}$ can be expressed in terms of relative orbital elements. In the keplerian case, given the vectors $\mathfrak{e}_{C}^{K}$ and $\mathfrak{e}_{D}^{K}$, the relative elements are simply computed as the difference between the elements of the two objects:

$$
\Delta \mathbf{e}^{K}=\mathfrak{e}_{D}^{K}-\mathbf{e}_{C}^{K}=(\Delta a \Delta e \Delta i \Delta \Omega \Delta \omega \Delta M)^{T} .
$$

In the non-singular case instead, the vectors $\boldsymbol{\propto}_{C}^{N}$ and $\mathfrak{\propto}_{D}^{N}$ lead to the relative elements through a nonlinear combination:

$$
\begin{aligned}
& \Delta \mathbf{e}^{N}=\left(\begin{array}{cccccc}
\Delta \widetilde{a} & \Delta \lambda & \Delta e_{x} & \Delta e_{y} & \Delta i_{x} & \Delta i_{y}
\end{array}\right)^{T} \\
& =\left(\begin{array}{cccccc}
\Delta a / a & \Delta u+\Delta \Omega \cos i & \Delta e_{x} & \Delta e_{y} & \Delta i & \Delta \Omega \sin i
\end{array}\right)^{T}
\end{aligned}
$$

where $\Delta \widetilde{a}$ is an additional measure of the differential semimajor axis, $\Delta \lambda$ is the differential mean longitude, $\Delta e_{x}$ and $\Delta e_{y}$ are the components of the relative eccentricity vector $\Delta \mathbf{E}=\left(\Delta e_{x} \Delta e_{y}\right)^{T}$ and finally $\Delta i_{x}$ and $\Delta i_{y}$ are the components of the relative inclination vector $\Delta \mathbf{I}=\left(\Delta i_{x} \Delta i_{y}\right)^{T}$. 


\section{Eccentricity/Inclination Vector Separation Technique}

The Eccentricity/Inclination (E/I) vector separation technique (EIVS) is particularly attractive since it can naturally enforce collision avoidance. It has been originally proposed to face the problem of satellites colocation in geostationary slots [11] and in the last years widely investigated and successfully applied also for formations of satellites in LEO ([8, 19, 7]).

Through the use of a rotating reference frame $C_{r \theta h}$ which has its origin coincident with the chief spacecraft, the $r \theta$-plane lying on its orbital plane and the $r$-axis parallel to its position vector (positive outwards), the relative motion can be expressed in non-dimensional form through:

$$
\begin{aligned}
& \delta x(u) \approx \Delta \widetilde{a} \quad-\Delta E \cos (u-\vartheta) \\
& \delta y(u) \approx-\frac{3}{2} \Delta \widetilde{a} u+\Delta \lambda+2 \Delta E \sin (u-\vartheta) \\
& \delta z(u) \approx+\Delta I \sin (u-\varphi)
\end{aligned}
$$

where $\Delta \mathbf{E}$ and $\Delta \mathbf{I}$ are expressed in polar notation, with $\Delta E=\|\Delta \mathbf{E}\|$ and $\Delta I=\|\Delta \mathbf{I}\|$ and with $\vartheta$ and $\varphi$ being the relative perigee and the relative ascending node [7].

Assuming $\Delta \lambda=0$ and $\Delta a=0$ to cancel the offsets and to prevent the drift in the along track direction, the collision risks can be reduced by setting:

$$
\begin{aligned}
\vartheta & =\varphi+k \pi \\
D_{\min } & \leq a \min \{\Delta E, \Delta I\}
\end{aligned}
$$

with $k$ integer, while the constraint

$$
a \sqrt{4 \Delta E^{2}+\Delta I^{2}} \leq D_{\max }
$$

ensures satisfaction of the MaxDC. If the $J_{2}$ perturbation is included in the model Eq. (4), $\Delta a=0$ is not valid anymore and Eqs. (5) and (6) need to be adapted [7].

To better relate the E/I relative vectors with the distances, it could be useful to consider their dimensional version obtained multiplying them with the semi-major axis of the VP. The new dimensional parameters can be distinguished by the original ones for the presence of a small hat ${ }^{(}($), so that to $\Delta E$ corresponds a $\Delta \widehat{E}=a \Delta E$, to $\Delta I$ corresponds a $\Delta \widehat{I}=a \Delta I$ and so on. Then, in the design phase, it can be helpful defining the elements of the spacecraft in the planes $\Delta \widehat{e}_{x} \Delta \widehat{e}_{y}$ and $\Delta \widehat{i_{x}} \Delta \widehat{i_{y}}$.

Let us now see how the EIVS approach can be used to configure a cluster with multiple objects. The problem can be geometrically faced in two steps:

1. in each of the two planes $\Delta \widehat{e}_{x} \Delta \widehat{e}_{y}$ and $\Delta \widehat{i_{x}} \Delta \widehat{i_{y}}$ the $D_{\max }$ is used to identify a region around the origin, which includes points satisfying the MaxDC;

2. in each region points are chosen with a mutual distance at least equal to $D_{\min }$.

An example of how the described geometric logic can be applied is given in Fig. 1, where each point represents the relative eccentricity (left plot) and the relative inclination (right plot) vector of a spacecraft w.r.t. the VP, which is highlighted as a small circle at the origin of the planes. 
Fig. 1 Example of relative eccentricity (left) and inclination (right) vectors for a cluster of 6 spacecraft.
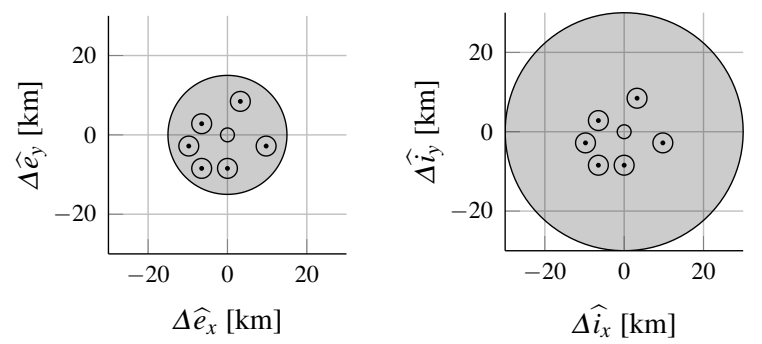

Provided that in each plane each point is sufficiently spaced from all the others and that corresponding points from the two planes satisfy the (anti-)parallelism condition (Eq. (5a)), the MinDC can be ensured. In the given example the MinDC are based on a value $D_{\min }=5 \mathrm{~km}$ and are represented by the small circles, inasmuch as they picture the forbidden regions around the spacecraft and, as it can be seen, are not overlapping. The fulfillment of the MaxDC can instead be studied by taking into consideration the distance of the points from the origin of the planes. These constraints are based on a value $D_{\max }=30 \mathrm{~km}$ and are represented by the gray regions, which can be evaluated through Eq. (4). It can be noted that in Fig. 1 corresponding points from the two planes, not only share the same phase of the relative vectors, but have also the same magnitude. This is the reason why in the right plot of Fig. 1 the points are crammed in the center leaving the outer part of the gray region empty. The condition of equal magnitudes is not required by the EIVS technique, but it has been introduced to limit the differential perturbations experienced by the spacecraft.

The given example shows how the relative E/I vectors can be selected while using simple 2-D geometry. Six points have been chosen from the gray regions but this number can easily grow if a smarter selection is performed. The maximization of $n_{m}$ becomes then a packing problem, since it turns into the research of the maximum number of points that can be placed into a given 2-D region. In this study the regular grid shown in Fig. 2 has been used, where each point is at the center of a regular

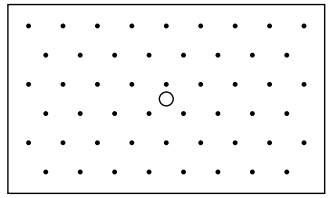

Fig. 2 Regular grid for the packing problem of the EIVS technique. hexagon and is surrounded by six points located at the vertices of the hexagon.

\section{$J_{2}$ Invariant Orbits Technique}

The technique of the $J_{2}$ invariant orbits (J2In) consists in placing the spacecraft in orbits that are invariant w.r.t. the effect of the $J_{2}$ term. When the $J_{2}$ perturbation is included in the motion of a spacecraft, its osculating elements experience oscillations and a secular growth. The oscillations are usually considered harmless, while the dangerous effect is the one associated with the secular growth, since it can produce a drift of the orbits. For this reason it can be useful focusing on the mean elements ${ }^{1}$ $\overline{\mathbf{a}}^{K}$, which are free from the oscillations and are only subject to the secular growth.

\footnotetext{
${ }^{1}$ Mean elements are usually identified by the presence of an overbar $\left(^{-}\right)$, but since the J2In tech-
} nique does not use osculating elements, to relieve the notation the overbar in this section is dropped. 
When the mean elements are used, it must be noted that the $J_{2}$ term only alters the elements $[\Omega \omega M]$, which experience time drifts modeled as [2]:

$$
\begin{aligned}
\frac{d \Omega}{d t} & =-\frac{3}{2} J_{2} n\left(\frac{R_{E}}{p}\right)^{2} \cos i \\
\frac{d \omega}{d t} & =-\frac{3}{4} J_{2} n\left(\frac{R_{E}}{p}\right)^{2}\left(5 \cos ^{2} i-1\right) \\
\frac{d M}{d t} & =n+\frac{3}{4} J_{2} n\left(\frac{R_{E}}{p}\right)^{2} \sqrt{1-e^{2}}\left(3 \cos ^{2}-1\right) .
\end{aligned}
$$

where $n=\sqrt{\mu / a^{3}}$ and $p=a \sqrt{1-e^{2}}$ are the mean motion and the semilatus rectum of the VP, while $\mu$ and $R_{E}$ are the gravitational parameter and the radius of the Earth.

The concept of the $J_{2}$ invariance is based on the fact that if the first three elements $\left[\begin{array}{ll}a & e\end{array}\right]$ of the different spacecraft are properly selected, it is possible to make them having the same rates of variation for the last three elements $[\Omega \omega M]$. Indeed this approach aims at canceling the relative rates, so that the secular drift can be prevented. Nevertheless, it is very uncommon to match all the three rates, because in this way the constraints would restrict considerably the possible relative orbits. It is instead usually preferred to combine the rate of $\omega$ and $M$ so that only the rates of $\dot{\Omega}$ and of $\dot{\omega}+\dot{M}$ need to be matched.

To perform the matching process, the constraints are expressed in terms of the deputy relative elements, hence the following expressions can be retrieved [24]:

$$
\begin{gathered}
f_{a}=\eta \Delta a+2 D a e \Delta e=0 \\
f_{i}=\eta^{2} \tan i \Delta i-4 e \Delta e=0
\end{gathered}
$$

where the absolute and differential elements used are the ones of the chief and of the deputy, respectively. In addition $\eta=\sqrt{1-e^{2}}, D=\frac{J_{2}}{4 L^{4} \eta^{5}}(4+3 \eta)\left(1+5 \cos ^{2} i\right)$ and $L=\sqrt{a / R_{e}}$ hold. Please note that Eq. (8) allows for computing $\Delta a$ and $\Delta i$ once that $\Delta e$ is fixed, but the cases where $\Delta a$ or $\Delta i$ are given remain also valid. Finally, for what concerns the remaining differential elements $[\Delta \Omega \Delta \omega \Delta M]$, they can be freely chosen as long as they satisfy the MinDC and the MaxDC conditions.

Let us now approach the problem for the cluster case. If multiple objects are involved in the process, the $J_{2}$ invariance should be ensured for all of them, meaning that each of their orbits should be $J_{2}$ invariant w.r.t. all the others. In this perspective Eq. (8) should be applied for each pair of elements (sat/VP and sat/sat), turning then into a set of $2 n_{p}$ conditions, with $n_{p}=0.5 n_{m}\left(n_{m}+1\right)$ denoting the number of the pairs. As soon as $n_{m}>2$ the system becomes overdetermined and needs then to be solved numerically, so that it becomes useful recasting Eq. (8) into the form

$$
\begin{aligned}
f_{a} & <\varepsilon \\
f_{i} & <\varepsilon
\end{aligned}
$$

where $\varepsilon$ is a user-defined threshold within which the solution must satisfy the constraints. According to the approximations used in the derivation of Eq. (8) (only 
terms of $\mathscr{O}\left(J_{2}\right)$ have been retained) [24], in the performed study is has been assumed $\varepsilon=10^{-3}$ to make it having the same order of magnitude of the $J_{2}$ coefficient.

Another step towards the definition of a cluster configuration consists in identifying the range of values from which the parameters $\Delta a, \Delta e$ and $\Delta i$ can be chosen. Given two objects $A$ and $B$ and stating that they move on $J_{2}$ invariant orbits, it should be implied that the orbit of $A$ is invariant w.r.t. the one of $B$ and viceversa. It has been seen instead, that if the differential elements [ $\Delta a \Delta e \Delta i]$ are too large, the above-mentioned double invariance can be missing. There are cases indeed where the elements of $B$ are selected to make it having an orbit invariant w.r.t. $A$, but then inverting the roles and treating $B$ as chief and $A$ as deputy, the invariance constraints are violated. To avoid this situation, the ranges of the differential elements able to ensure the desired double invariance have been researched. According to the eccentricity of the VP, the attention has been posed on an interval $-0.009<\Delta e<0.039$, which has then been divided into a regular grid. Next, for all the grid points the double invariance has been pairwise checked and the results are depicted in Fig. 3. The gray area emphasizes the satisfaction of the double invariance, which for example is fulfilled by two objects having orbital elements defined with differential eccentricities equal to $2.7 \times 10^{-2}$ and $-0.3 \times 10^{-2}$, but not for those two with orbital elements obtained from the values $3.9 \times 10^{-2}$ and $1.5 \times 10^{-2}$. Please note that the plot is symmetric w.r.t. the plan bisector and therefore for both the given examples, the results do not depend on which of the two values is assigned to which of the two objects. Finally the black-sided square highlights the wanted

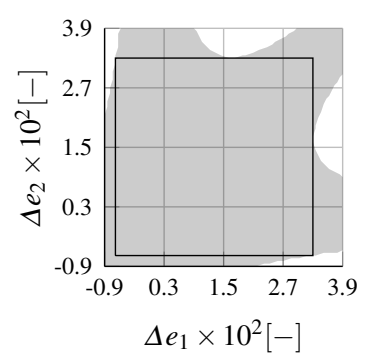

Fig. 3 Map of the $J_{2}$ double invariance shown in terms of relative eccentricities. range for the differential eccentricity, so that if all the orbits of the cluster satisfy

$$
\begin{aligned}
-0.023 \mathrm{~km} & <\Delta a<0.048 \mathrm{~km} \\
-0.007 & <\Delta e<0.033 \\
-0.00025 \mathrm{rad} & <\Delta i<0.00120 \mathrm{rad}
\end{aligned}
$$

they are all $J_{2}$ invariant w.r.t. each other.

To investigate the cluster case, the definition of the initial configuration can be expressed as the determination of those vectors $\Delta \mathfrak{\varkappa}_{i}^{K}$ (with $i=1,2, \ldots, n_{m}$ ), whose elements are bounded by the conditions given in Eq. (10) and also fulfill a system of nonlinear conditions given by the $2 n_{p}$ equations obtainable from Eq. (8) and by additional equations deriving from the application of Eq. (1). To research the solution the solvers FMINCON from MATLAB and SNOPT have been used.

In the way the problem has been implemented, it is trivial that $n_{m}$ is not a variable, but a parameter provided by the user. Therefore, to maximize the number of spacecraft that the cluster can allocate, it has been decided to proceed manually solving the problem with different increasing values of $n_{m}$. When a solver cannot find a solution anymore, the cluster is saturated and the maximum value for $n_{m}$ has been found. 


\section{Distance Bounded Natural Orbits Technique}

The technique of the distance-bounded natural orbits (DBNO), as suggested by the name itself, aims at finding orbits that satisfy naturally the MinDC and the MaxDC. The technique is presented in Mazal and Gurfil [17] and relies on a constraint which is proven to ensure bounded relative distances when a time invariance assumption for the environmental perturbations is made. This idea is similar to what is done with the J2In approach, but this time the invariance can take all perturbations deriving from the gravitational potential into account.

Given two objects $A$ and $B$ with equal ballistic coefficients, if it results

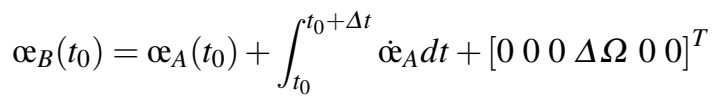

then it also holds

$$
2 \eta_{\min } \sin \left(\frac{|\Delta \Omega|}{2}\right)-V_{\text {max }}|\Delta t| \leq d_{s}(t) \leq 2 \eta_{\max } \sin \left(\frac{|\Delta \Omega|}{2}\right)+V_{\text {max }}|\Delta t|
$$

where $V_{\max }$ denotes the maximum speed of $A$, while $\eta_{\max }$ and $\eta_{\min }$ denote its maximum and minimum equatorial projections, being $\eta_{A}(t)=\sqrt{x_{A}^{2}(t)+y_{A}^{2}(t)}$.

For a given set of constraints on the minimum and maximum distances $D_{\text {min }}$ and $D_{\max }$, the values $\Delta t$ and $\Delta \Omega$ can be chosen while satisfying

$$
\begin{gathered}
2 \eta_{\min } \sin \left(\frac{|\Delta \Omega|}{2}\right)-V_{\text {max }}|\Delta t| \geq D_{\text {min }} \\
2 \eta_{\max } \sin \left(\frac{|\Delta \Omega|}{2}\right)+V_{\text {max }}|\Delta t| \leq D_{\max }
\end{gathered}
$$

Equation (13) can be observed graphically in Fig. 4, showing for which values of $\Delta \Omega$ and $\Delta t$ the MinDC and the MaxDC are satisfied, when $D_{\min }=5 \mathrm{~km}$ and $D_{\max }=100 \mathrm{~km}$ are considered. The satisfaction of each constraint is highlighted with a different color, therefore for the mission design a pair $[\Delta \Omega \Delta t]$ must be selected from the overlapping area.

In the case of $n_{m}$ spacecraft, the DBNO technique keeps being valid as long as the constraints expressed through Eq. (13) are applied to each pair of them. The differences in time and

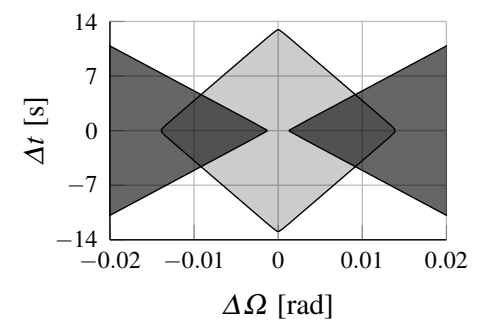

Fig. 4 Example of $\Delta t$ and $\Delta \Omega$ selection while satisfying the MinDC ( $\square)$ and the $\operatorname{MaxDC}(\square)$.

RAAN of the $i$-th spacecraft w.r.t. the VP can be denoted as $\Delta \Omega_{0 i}$ and $\Delta t_{0 i}$, while the differences between any pair of spacecraft $(i, j)$ can be denoted as

$$
\left\{\begin{array}{l}
\Delta \Omega_{i j}=\Delta \Omega_{0 j}-\Delta \Omega_{0 i} \\
\Delta t_{i j}=\Delta t_{0 j}-\Delta t_{0 i}
\end{array} \quad \text { with } j=1,2, \ldots, n_{m} \text { and } j \neq i .\right.
$$


In addition, the small angles approximation can be introduced to replace the sine functions with their arguments. Indeed in this work several $D_{\max }$ up to few hundreds of $\mathrm{km}$ will be considered, leading to a maximum differential RAAN of few degrees totally compatible with this approximation. Equation (13) turns then into:

$$
\begin{aligned}
\eta_{\min }\left|\Delta \Omega_{i j}\right|-V_{\max }\left|\Delta t_{i j}\right| & \geq D_{\min } \\
\eta_{\max }\left|\Delta \Omega_{0 i}\right|+V_{\max }\left|\Delta t_{0 i}\right| & \leq D_{\max }
\end{aligned}
$$

Concerning Eq. (15b) and the MaxDC, it should be noted that the hypothesis of equal ballistic coefficients is missing in this work, since the VP is not a real spacecraft and cannot be affected by the atmospheric drag. This means that a slow drift of the spacecraft w.r.t. the virtual point should be expected.

When approaching the problem while trying to maximize the number of modules $n_{m}$, Eq. (15b) is immediately used to define the domain of the differential time and RAAN from which the sets $\left[\Delta \Omega_{0 i} \Delta t_{0 i}\right]$ should be selected. On the other side Eq. (15a) is exploited for the actual selection of the sets $\left[\Delta \Omega_{0 i} \Delta t_{0 i}\right]$. From a first glimpse at Eq. (15a) and with the help of Fig. 4, it can be seen that it is never possible to satisfy the MinDC with a pure time shift and that a minimum differential RAAN $|\Delta \Omega|_{\min }$ is always required. Indeed exploiting Eq. (15a) and the fact that $\left|\Delta t_{i j}\right|>0$, the minimum value $|\Delta \Omega|_{\min }$ can be retrieved

$$
\left|\Delta \Omega_{i j}\right|>\frac{D_{\min }}{\eta_{\min }} \equiv|\Delta \Omega|_{\min }
$$

and the differential RAAN can be chosen according to $\left|\Delta \Omega_{i j}\right|=\left(1+k_{\Omega}\right)|\Delta \Omega|_{\text {min }}$ with a small $k_{\Omega}>0$. In turn an upper bound for the time shift can be defined as

$$
\left|\Delta t_{i j}\right|<\frac{k_{\Omega} D_{\min }}{V_{\max }} \equiv|\Delta t|_{\max }
$$

and similarly to what has been set for the differential RAAN, the differential times can be chosen according to $\left|\Delta t_{i j}\right|=\left(1-k_{t}\right)|\Delta t|_{\max }$ with a small $k_{t}>0$. It is worth noticing that the determination of the two boundaries $|\Delta \Omega|_{\min }$ and $|\Delta t|_{\max }$ and the selection of the two coefficients $k_{\Omega}$ and $k_{t}$ do not depend on the specific pair $(i, j)$, therefore it is possible to define the values of the four parameters just once and use them to find the initial condition of all the spacecraft.

At this point, it is clear that dividing the $\Delta \Omega$ domain in a grid of points equally spaced by $\left(1+k_{\Omega}\right)|\Delta \Omega|_{\min }$ allows finding the differential RAAN $\Delta \Omega_{0 i}$ and maximizing the number of spacecraft $n_{m}$ at the same time. Concerning the time shifts, a trivial solution can be obtained assuming $\Delta t_{0 i}=0 \forall i$. Otherwise if time shifts different from zero are desired they can be selected through the knowledge of the $|\Delta t|_{\max }$.

\section{Delayed Elements Technique}

The technique of the delayed elements (DeEl) is very similar to the DBNO technique, inasmuch as it aims at finding relative orbits which are invariant w.r.t. the perturbations deriving from the full gravitational potential. The main difference con- 
sists in the fact that the elements of the spacecraft in this case are obtained only through the use of the time difference $\Delta t$, which means that all the members of the cluster pass through the same positions of the virtual point, but they do it with a certain time difference $\Delta t$ [9]. In this way all the spacecraft experience the same perturbations with minimal variations from the gravitational field with the consequence that maneuvers to counteract differential perturbations are greatly reduced.

The cluster obtainable with this technique can be imagined as a train of spacecraft separated in the along track direction, just like pearls on a string. Talking about separation distance $d$ or time difference $\Delta t$ is equivalent, since these two quantities can be easily related exploiting the knowledge of the mean motion. Indeed given a spacecraft with mean motion $n$ moving for a time $\Delta t$, the distance $d$ between the initial and the final positions can be approximated with the traveled arc of trajectory $\widehat{d}$, which exploiting Kepler's second law can be expressed as

$$
\widehat{d}=\frac{a b n \Delta t}{r}
$$

where $b$ is the semiminor axis of the orbit and $r$ is the position vector magnitude. It is worth noticing that for an elliptical orbit $r$ changes with time and according to the location of the spacecraft along the orbit a different $\bar{d}$ can correspond to the same $\Delta t$. For this reason once that the maximum and the minimum values of $r$ are computed, the minimum and the maximum distances corresponding to the given $\Delta t$ are also known. From a different point of view, this means also that when the $D_{\min }$ and $D_{\max }$ values are given, the evaluation of the required time difference $\Delta t$ is quite straightforward. And this is exactly how the initial conditions of two spacecraft can be defined, since in this case the $D_{\min }$ and $D_{\max }$ are assumed to be known and can be used to retrieve a range of values from which the $\Delta t$ should be picked to satisfy the distance constraints. Once that the $\Delta t$ is chosen, the initial state of a spacecraft $B$ can be computed from that of a spacecraft $A$ through Eq. (11), assuming $\Delta \Omega=0$.

In a similar way, the configuration of an entire cluster can be approached, with the core of the process consisting in the identification of the time shifts associated with the different objects of the cluster. In this perspective, following the example of the DBNO technique, it can be useful to distinguish between the time shifts of the spacecraft w.r.t. the VP $\Delta t_{0 i}$ and the time shifts among the spacecraft

$$
\Delta t_{i j}=\Delta t_{0 j}-\Delta t_{0 i}
$$

with $i, j=1,2, \ldots, n_{m}$ and $i \neq j$. The constraints on the distances only appear indirectly, inasmuch as they are used to define an upper and a lower bound for the values of the time shifts $\Delta t_{0 i}$, which need to be researched while satisfying Eq. (19). In particular, the MaxDC defines the maximum allowed time shift $|\Delta t|_{\max }$ of a spacecraft w.r.t. the VP, meaning that it should result $\frac{\Delta t_{0 i}}{|\Delta t|_{\max }} \in\left[\begin{array}{ll}-1 & 1\end{array}\right]$. On the other side the MinDC is taken into account computing the minimum time shift $|\Delta t|_{\text {min }}$ corresponding to the minimum distance $D_{\min }$ and ensuring that $\left|\Delta t_{i j}\right|>|\Delta t|_{\min } \forall(i, j)$. 
Finally, when the interval $\left[-|\Delta t|_{\max }|\Delta t|_{\max }\right]$ is divided into sub-intervals of length $|\Delta t|_{\text {min }}$, the problem of maximizing the number of spacecraft $n_{m}$ is also addressed, with the nodes of the grid denoting the time shifts $\Delta t_{0 i}$.

\section{Comparison of the Techniques}

To analyze the behaviour of the different techniques and perform their comparison, different points of view have been considered focusing on the number of spacecraft populating the cluster and on the $\Delta V$ they require for the corrective maneuvers.

The maximum number of points $n_{m}$ that can be packed into a given volume of space around the VP clearly depends on the constraints MinDC and MaxDC, since for a given value of $D_{\min }$ an increase of $D_{\max }$ involves a larger volume around the VP with the chance to allocate more points in it, while for a given value of $D_{\max }$ an increase of $D_{\min }$ involves a larger safety distance between any pair of points with a consequent reduction of their total number for that same volume. This makes then $n_{m}$ dependent on three different aspects of the problem: the TIC, the $D_{\text {min }}$ and the $D_{\max }$. To observe the effect of the distances, the constraints given in Eq. (1a) and Eq. (1b) have been applied with several values of $D_{\min }$ and $D_{\max }$. For the sake of brevity, in the following a specific set of values $\left[D_{\min } D_{\max }\right]$ might also be denoted simply as $D_{R}=D_{\min } / D_{\max }$ so that, for example, the notation $D_{R}=1 / 10$ indicates that the cluster needs to satisfy a $D_{\min }=1 \mathrm{~km}$ and a $D_{\max }=10 \mathrm{~km}$.

Concerning the $\Delta V$, it is clear that due to the differential perturbations experienced, the spacecraft will naturally drift over time and even if the cluster is initialized to satisfy the distance constraints, sooner or later the relative configuration might become unsafe. The safety of the cluster can be ensured through proper corrective maneuvers, hence the required $\Delta V$ can be used as second key parameter.

\subsection{Slots vs Spacecraft}

This section it intended to shortly explain that a distinction between the slots occupied by the spacecraft and the spacecraft themselves has been made.

The slots are those reference locations in which the spacecraft are deployed and that should be tracked by them. They are the entities directly involved in the search process and are treated as mass-less points. The ultimate goal is to find locations that satisfy the distance constraints indefinitely, so that when the spacecraft are deployed in them and track them through station keeping maneuvers, one can be sure that the distance constraints will not be violated. Due to this distinction, in the early phase of the study, for each set [TIC $D_{\min } D_{\max }$ ], the TIC is used to identify slots that ensure satisfaction of the MinDC and the MaxDC. Then, in a second moment the slots are filled with spacecraft and their motion is studied. 


\subsection{Number of Slots}

The identification of the slots consists in defining the initial reference vectors while taking also their natural evolution into account. A set of states satisfying the constraints at time $t=0 \mathrm{~s}$ and violating them at $t=10 \mathrm{~s}$ would be as useless as one violating them at $t=0 \mathrm{~s}$. An ideal set never violates the constraints, but this is not a realistic case because of the differential perturbations, which bring the vectors to slowly drift towards an unsafe configuration. The drifts grow indeed over time involving an increase in the number of violations. What can be done is to treat the configuration returned by the TIC as a candidate solution, propagate it for a certain timeframe and discard the state vectors violating the distance constraints.

For each set [TIC $D_{\min } D_{\max }$ ] a certain number of candidate slots $n_{s, c}$ is obtained with the initial state of each slot defined as:

$$
\mathbf{e}_{s}\left(t_{0}\right)=f\left(\mathbf{e}_{V P}\left(t_{0}\right)+\Delta_{s}\right)
$$

with $s=1,2, \ldots, n_{s, c}$ and $f$ is a function denoting the fact that the state of the $s_{t h}$ slot is obtained applying some differences $\Delta_{s}$ to the initial state of the virtual point. In Eq. (20) a generic function $f$ has been used to take into account that those differences change from one technique to another. They can be the differential time $\Delta t$ of the DeEl technique, or the differential mean elements of the J2In technique, and so on.

Once that the states of the candidate slots are available, they are converted in Cartesian coordinates and propagated for five orbits including the $J_{2}$ effect. The choice for such a short time frame is due to the fact that some techniques (EIVS, J2In) identify the initial configuration by using simplified models of the relative motion, which become less and less reliable over time. The decision to include the $J_{2}$ perturbation is instead motivated considering that its effect is the largest one in a LEO. A spacecraft would also be significantly perturbed by its interaction with the atmosphere, but in this first phase the main characters of the study are the slots, which can be treated as mass-less points free from the effect of the drag.

Once that the Cartesian propagation is completed the relative distances are recovered and checked. At first the check of the maximum distance from the virtual point is considered and the slots violating it are directly discarded from the solution. Afterwards the attention shifts towards the MinDC, whose check takes advantage of the graph theory $([10,1])$. It is clear that a slot violating the MaxDC cannot be part of the solution, but if the slots $(x, y)$ violate the minimum distance there is no need to remove them both, rather is it enough to discard only one of them and two solutions may be used, i.e. one including $x$ without $y$ and another including $y$ without $x$. However, as the number of MinDC violations increases, it becomes more and more difficult to identify the slots to remove; hence the graph theory might come in handy. In particular, a graph can be constructed from all the pairs of slots satisfying the minimum distance constraints, so that the identification of the most populated cluster can be set as a maximum clique problem MCP $([4,28])$.

For the sake of clarity let us consider a practical example with five candidate slots all satisfying the MaxDC. Suppose instead that a violation of the minimum distance is recorded for the pairs of slots $(1,2),(1,5)$ and $(2,3)$. Solving the MCP for the 
graph associated with the problem, three maximum cliques can be found:

$$
C_{1}=\left\{\begin{array}{lll}
13 & 4
\end{array}\right\} \quad C_{2}=\left\{\begin{array}{ll}
245 \\
2
\end{array} \quad C_{3}=\{345\}\right.
$$

This means that given the five candidate slots, the largest safe cluster cannot include more than three of them, and moreover not every three-element combination of the five candidates is acceptable, rather only the combinations $C_{1}, C_{2}$ and $C_{3}$.

The combinations of slots obtained from the MCP resolution represent then solutions of the initial configuration problem and once that they are available the search can be considered concluded. Figure 5 shows how the number of slots varies with the $D_{\max }$ for all the techniques examined and for $D_{\min }$ fixed at $1 \mathrm{~km}, 5 \mathrm{~km}, 9 \mathrm{~km}, 13$ $\mathrm{km}$ and $17 \mathrm{~km}$. As expected, for every TIC, the number of returned slots increases when increasing $D_{\max }$ once the $D_{\min }$ is given and decreases when increasing $D_{\min }$ once the $D_{\max }$ is given. The less populated clusters would be the ones obtained from the DBNO, whose trend is always the lowest. A slight increase in the slots number is achieved with the J2In and DeEl, which behave similarly, with trends very close to each other. Finally the EIVS is the technique returning the largest slots number in most of the cases, with the separation between its and the rest of the trends getting larger when reducing the minimum distance boundary. This behaviour depends on the fact that the constraints required by the EIVS are less restrictive than those imposed by the other techniques, with the consequence that a larger number of vectors satisfying them can be found.
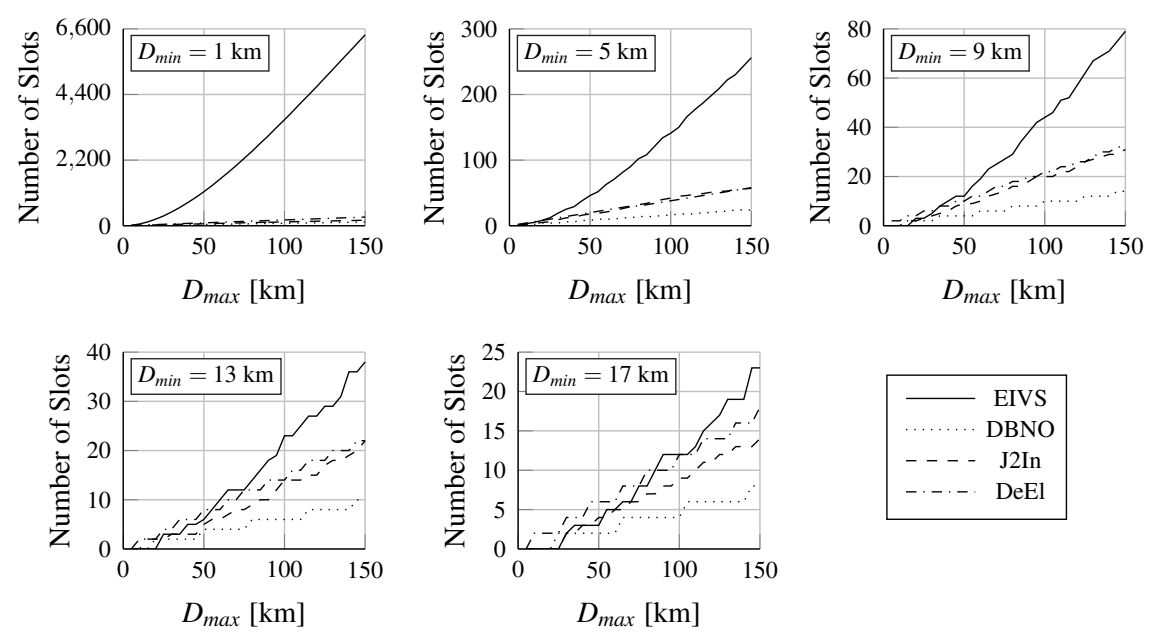

Fig. 5 Number of slots as a function of $D_{\max }$ for the different techniques.

Graphically Fig. 6 can also help, giving an overview of the arrangement associated with the different techniques. Each subfigure is associated with a TIC and shows the initial configuration of slots around the VP when the distance boundaries are given by $D_{R}=17 / 85$. The representation is given in an inertial frame translated to shift its origin in the virtual point, which is then located at the center of the subfigures and colored in black, just like the solid lines representing its orbit. As it can 
be seen, the slots from EIVS are densely arranged on a plane, while the slots from the DBNO and DeEl are arranged on a line and the slots from the J2In are sparsely distributed in a 3-D region.

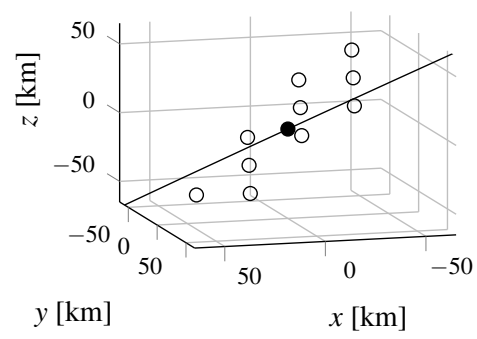

(a) EIVS, 10 slots

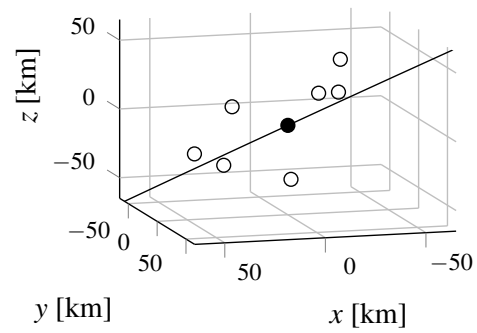

(c) J2In, 7 slots

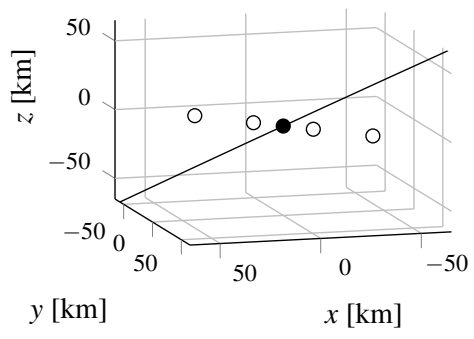

(b) DBNO, 4 slots

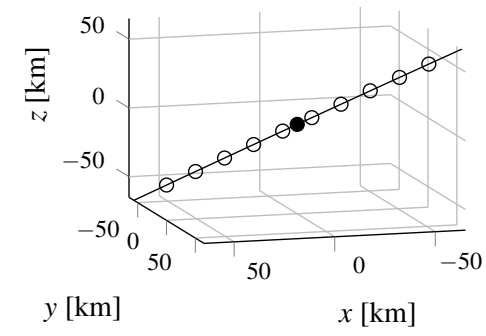

(d) DeEl, 10 slots

Fig. 6 Slots arrangements returned by all techniques for $D_{\min }=17 \mathrm{~km}$ and $D_{\max }=85 \mathrm{~km}$.

\section{$3.3 \Delta V$ Budget}

Once that the study of the slots had been completed, the attention has been shifted towards the behaviour of the spacecraft, with the final goal of evaluating the $\Delta V$ budget required to maintain the distance constraints satisfied and the cluster safe. As a matter of fact, the maximum number of spacecraft that can be packed into a cluster is for sure an important parameter to be considered for a cluster mission, but at the same time one cannot forget the key role played by the $\Delta V$ budget.

To perform this type of analysis, several sample slots configurations have been selected and for each configuration simulations have been run assigning a spacecraft to each of its slots. In particular, a direct correspondence has been implemented so that each spacecraft is assigned to a single slot and tries to track it using station keeping maneuvers. Each simulation consisted in a propagation of the cluster initial configuration in an inertial frame under the effect of the gravitational potential (terms up to $J_{20}$ have been included) and of the drag. The different TIC examined try to counteract the differential perturbations in a different way, hence it is reasonable to expect a different evolution of the slots over time and a different amount of $\Delta V$ required for the station keeping. 
The station keeping has been thought as composed by three phases. At first the spacecraft computes the offset, in terms of differential orbital elements, of its current state from its reference state. Then the maneuver to cancel the offset is computed as a sequence of impulsive control actions aimed at correcting the orbital elements. Finally the maneuver is executed. Please note that in the second phase each spacecraft computes the maneuver taking only its own elements into account. The relative motion w.r.t. the other spacecraft is entirely neglected, hence during the execution of the control actions small violations of the distance boundaries might be recorded.

For this study, five sample configurations have been observed, one for each value of the $D_{\min }$ parameter. The maximum distance constraints are instead based on a value $D_{\max }=5 D_{\min }$. Table 2 summarizes the five cases showing the number of spacecraft $n_{m}$ for the four techniques.

Table 2 Number of spacecraft obtainable for five sample cluster configurations.

\begin{tabular}{ccccc}
\hline$D_{R}$ & EIVS & DBNO & J2In & DeEl \\
\hline $1 / 5$ & 14 & 4 & 4 & 10 \\
$5 / 25$ & 12 & 4 & 6 & 10 \\
$9 / 45$ & 12 & 4 & 8 & 10 \\
$13 / 65$ & 12 & 4 & 7 & 10 \\
$17 / 85$ & 10 & 4 & 7 & 10 \\
\hline
\end{tabular}

For each examined configuration several tests have been performed, since it has been decided to implement a different logic to trigger the station keeping maneuvers:

PM - a fixed periodic maneuvering cycle is implemented, i.e. at regular time intervals all the spacecraft measure their offsets, compute the corrective maneuver to cancel them and perform it;

$\mathrm{CV}$ - each spacecraft computes and performs the required maneuvers only when the violation of one of the constraints MinDC or MaxDC is detected.

The PM logic has the advantage that it allows to know a priori the times at which the computation of the maneuver is performed, but as a drawback the constraints on the distances do not play any role and if a violation occurs nothing is done to fix it. Conversely, in the CV logic the distance constraints are actively used and only the involved spacecraft actually perform a maneuver, but as it will be seen exploring the results, it can happen that some spacecraft require very sparse but large corrections, that can produce severe violations of the constraints.

One parameter for the evaluation and comparison of the results can be identified in the average $\Delta V$ required by the cluster, i.e. the total $\Delta V$ required by all the spacecraft averaged w.r.t. $n_{m}$. This choice is suggested by the fact that the number of spacecraft changes for each of the four techniques and usually also with the selected maneuver logic, hence the comparison of the total $\Delta V$ would make no sense.

Figures 7 and 8 compare the results for the initial configuration obtained with the technique EIVS for $D_{R}=1 / 5$. The considered time horizon is 10 days. In Fig. 7 the $\Delta V$ budget is shown while Fig. 8 gives an overview of the number of the required 


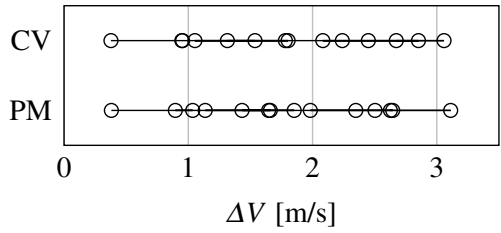

Fig. $7 \Delta V$ budget required by an initial configuration from the technique EIVS for two maneuver logics.

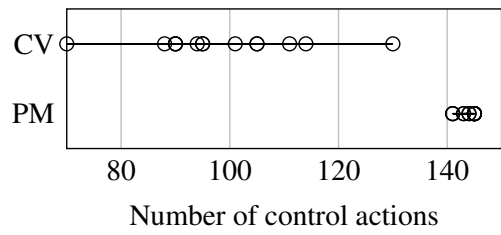

Fig. 8 Number of control actions required by an initial configuration from the technique EIVS for two maneuver logics.

control actions. In both plots a single circle refers to a single spacecraft of the cluster. The spreading of the circles for a given maneuver logic depends on the fact that according to the distance between a spacecraft and the VP, the differential perturbations experienced are different and then a different amount of $\Delta V$ is required. With particular reference to Fig. 8, when the CV maneuver logic is implemented the different differential perturbations bring the spacecraft to violate the distance constraints at different times, that is why the circles are so spread. On the other side, when the PM logic is used, all the spacecraft perform the corrective maneuvers periodically, hence they all require approximately the same number of control actions. It can then be summarized that for the shown example the maneuver logic does not have a big impact on the average $\Delta V$, rather it affects the number of control actions used and the way the propulsion system is stressed. As a matter of fact, an increase in the time interval between two maneuvers implies larger sparse maneuvers instead of shorter frequent ones.

Table 3 gives instead a measure of the constraints violations recorded in the considered example showing the minimum and the maximum distances reached.

Table 3 Distance violations detected by an initial configuration from the technique EIVS for two maneuver logics.

\begin{tabular}{lcc}
\hline Logic & $D_{\min }$ & $D_{\max }$ \\
\hline PM & $6.73 \%$ & $1.22 \%$ \\
CV & $21.18 \%$ & $2.08 \%$ \\
\hline
\end{tabular}

The use of percentage values stems from the comparison of the maximum violations with the foreseen boundaries set at $D_{\min }=1 \mathrm{~km}$ and $D_{\max }=5 \mathrm{~km}$. The MaxDC violations are on the same order of magnitude for both the maneuver schemes PM and $\mathrm{CV}$, meaning that in both cases the maximum distance reached is up to approximately $2 \%$ larger than the $5 \mathrm{~km}$ boundary value. A significant difference can be seen instead on the MinDC side, since in the PM case the minimum distance reached stays above $93 \%$ of the $1 \mathrm{~km}$ boundary value, while in the CV case it can decrease until $78 \%$ of it. It must be pointed out that such constraints violations occur sporadically and always within the first one or two orbits after the execution of the first control action, but this does not mean that the station keeping strategy does not work. This behaviour was already expected and can be attributed to the particular way the trajectory corrections have been implemented. As a matter of fact the station keeping is not instantaneous, but is composed by several separate impulsive control actions, taking place at different locations along the orbit. Between the first 
and the last control action the spacecraft travel on transfer orbit arcs and it is in this short timeframe that the violations are recorded. Indeed, as it can be recalled from the description of the three phases of the station keeping, for each spacecraft the required maneuvers are computed to make it track its reference state, but without taking into consideration the relative motion w.r.t. the other spacecraft during the execution of the maneuver itself.

Despite the fact that the $\mathrm{CV}$ control logic actually requires a violation of the distance boundaries to trigger the computation of a correcting maneuver, in general a violation is clearly undesirable. Nevertheless in this part of the study the intention was not really to prevent violations as a whole, rather to see if a station keeping approach could be sufficient to perform cluster keeping and to obtain a raw evaluation of the $\Delta V$ budget required by different configuration designs.

In Figs. 9 and 10 an overview of the violations is depicted for the MinDC and the MaxDC, respectively. Each figure is divided into four parts in order to compare the different techniques. Each part illustrates the behaviour of a TIC through two bars, one for each of the two tested maneuver logics. Recalling that for a single TIC and a single maneuver logic five sample configurations have been tested, each bar renders the worst out of the five cases, i.e. the largest percentage violation w.r.t. the reference distance boundary. For example if one considers the EIVS technique and a station keeping strategy applied regularly every five orbits, it can be seen that the maximum violations of the MinDC and the MaxDC are below 10\% and 5\%, respectively.

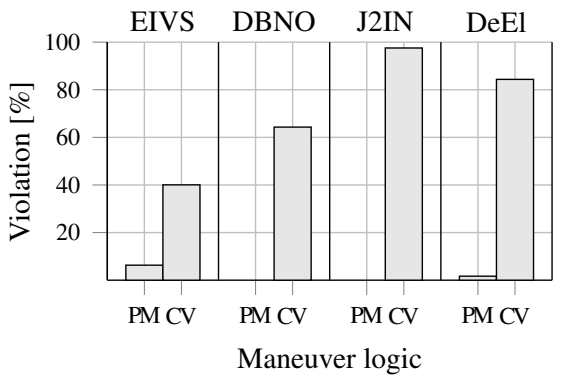

Fig. 9 Violations overview of the MinDC.

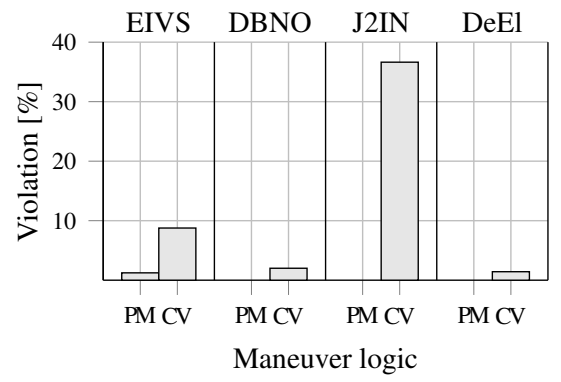

Fig. 10 Violations overview of the MaxDC.

Observing the different parts of the figures singularly, it can be noticed that the $\mathrm{CV}$ bar is always higher than the PM bar. This trend depends on the fact that, as already mentioned, when the timeframe between two consecutive maneuvers increases, the differential drift of the spacecraft from the path of their reference initial states increases as well, so that larger correcting maneuvers are required and larger violations can be experienced. In addition, in the $\mathrm{CV}$ cases the bars can become much higher than the PM cases, because a maneuver already starts with a violation, which then is accentuated by the application of the corrections.

As a final outcome, it is clear that the station keeping framework is not entirely violations-free and that the relative motion during the transfer paths should also be taken into consideration. If this aspect of the problem was implemented, the control 
strategy would have been different, with a different sequence of control actions but without a substantial change in the overall estimated $\Delta V$ budget.

To conclude this part of the study and the comparison of the different techniques, let us now focus on the results of the station keeping investigation in terms of the $\Delta V$ required by the spacecraft. So far it has been seen that the different TIC provide a different number of slots and react differently to the distance constraints, but a comparison of the $\Delta V$ budget is still missing.

To develop this analysis, two points of view can be considered. Let us focus on a single $D_{R}$. On one side one can fix the maneuver logic and pay the attention on entire configurations. On the other side it could be interesting to fix a maximum $\Delta V$ budget and see, for each TIC, how many spacecraft comply with this limitation. For a better understanding, Figs. 11 and 12 come in handy, respectively.

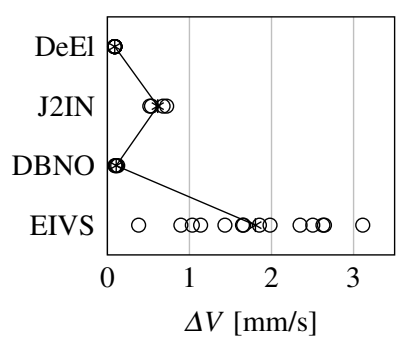

Fig. $11 \Delta V$ budget for clusters obtained with different TIC (maneuver logic PM and case $D_{R}=1 / 5$ ).

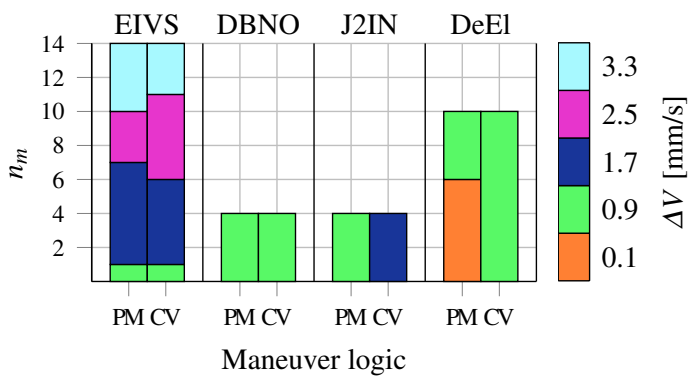

Fig. 12 Number of deployable spacecraft according to different $\Delta V$ thresholds for different TIC and different maneuver logics, in the case $D_{R}=1 / 5$.

Figure 11 focuses on the case $D_{R}=1 / 5$ with station keeping performed every five orbits. Each circle denotes the $\Delta V$ required by a spacecraft during the entire time frame of 10 days, while the stars and the solid line connecting them highlight the average $\Delta V$ required by the entire cluster. As expected, the less differential perturbations are included in the definition of the initial configuration, the larger the drift among the spacecraft is and the less homogeneous the fuel consumption is. That is why for the DeEl and DBNO the $\Delta V$ of the single spacecraft are very close to the average value, while the spreading increases for the EIVS and the J2In.

The second type of research led instead to Fig. 12, which refers again to the case $D_{R}=1 / 5$, but this time all the TIC and the maneuver logics are included. In this analysis it has been decided to seek how many spacecraft could be deployed from the different investigated clusters, when a constraint on the maximum available $\Delta V$ is set. For the case given in Fig. 12 five threshold values are considered. It can be observed for example that if the smallest threshold is selected, only spacecraft consuming less than $0.1 \mathrm{~mm} / \mathrm{s}$ can be used, which means that only one configuration is available, i.e. a six-objects cluster from the DeEl technique performing station keeping every five orbits. If the threshold is raised to $0.9 \mathrm{~mm} / \mathrm{s}$, some solutions from the DBNO and J2In become also available, while to use a cluster from the EIVS a threshold of $1.7 \mathrm{~mm} / \mathrm{s}$ is required. Similar results are obtained from the other $D_{R}$, 
with the DeEl always showing a good compromise of high number of spacecraft and low $\Delta V$ budget, followed by the DBNO, the J2In and finally the EIVS. On the other side the EIVS can always provide the most populated clusters, but the price for this asset is paid in terms of high $\Delta V$ requirements.

\section{Conclusions}

Exploiting the concept of fractionation it is possible to split and replace a single monolithic spacecraft with multiple smaller ones flying close to each other. In such a framework a safe relative motion must be guaranteed, bounding the minimum and the maximum relative distances to prevent, respectively, collisions and escaping drifts. With a proper orbit design, the spacecraft might initially satisfy the relative motion constraints, but due to the differential perturbations acting on them, over time their configuration slowly becomes unsafe and corrective maneuvers need to be performed. In this work several designs have been surveyed, each providing a cluster initial configuration while counteracting the differential perturbations with a different approach. The identified test setup assumes that: 1) the cluster is centered in a virtual point; 2) a minimum distance must be ensured between any pair of spacecraft to prevent collisions; 3) a maximum distance must be ensured between any spacecraft and the virtual point to prevent escaping drifts; 4) each spacecraft tracks a reference state through station keeping maneuvers.

In the first part of the study, it has been investigated how the number of deployable spacecraft changes according to the design and to the minimum and maximum distance constraints. Later on, the obtained initial configurations have been propagated in time and station keeping maneuvers have been implemented to evaluate the $\Delta V$ budget required by the single spacecraft and the entire clusters.

It emerged that in the design phase several aspects of the problem need to be taken into account, like the number of deployable spacecraft, the need to introduce countermeasures against violations of distance boundaries, the differential consumption of fuel, etc. All these aspects are clearly interconnected with each other and it is not possible to identify a configurations which has the best performances from all points of view. According to the specific requirements of the mission the best fitting configuration can be different, but the conducted study provides an indication and an analysis method.

Acknowledgements This research has been funded by the German Israeli Foundation Grant No. 1181-220.10.

\section{References}

[1] V. Balakrishnan. Schaum's Outline of Theory and Problems of Graph Theory. Schaum's Series. McGraw-Hill Education, 1997.

[2] R. Battin. An Introduction to the Mathematics and Methods of Astrodynamics, Revised Edition. Ed. by J. S. Przemieniecki. American Institute of Aeronautics \& Astronautics, 1999. 
[3] I. Beigelman et al. "Optimal Fuel-Balanced Impulsive Formationkeeping for Perturbed Spacecraft Orbits". In: Journal of Guidance, Control, and Dynamics 31.5 (2008).

[4] R. Carraghan et al. "An exact algorithm for the maximum clique problem". In: Operations Research Letters 9.6 (1990).

[5] T. Carter et al. "Fuel-Optimal Rendezvous Near a Point in General Keplerian Orbit". In: Journal of Guidance, Control, and Dynamics 10.6 (1987).

[6] W. Clohessy et al. "Terminal Guidance System for Satellite Rendezvous". In: Journal of the Aerospace Sceinces 27.9 (1960).

[7] S. D’Amico. "Autonomous Formation Flying in Low Earth Orbit". PhD thesis. Delft, Netherlands: Technical University of Delft, 2010.

[8] S. D'Amico et al. "Proximity Operations of Formation-Flying Spacecraft Using an Eccentricity/Inclination Vector Separation”. In: Journal of Guidance, Control, and Dynamics 29.3 (2006).

[9] F. J. de Bruijn et al. "Delayed Target Tracking for Along-Track Formations". In: Journal of Guidance, Control, and Dynamics 38.7 (2015).

[10] R. Diestel. Graph Theory. Electronic library of mathematics. Springer, 2000.

[11] M. Eckstein et al. "Colocation Strategy and Collision Avoidance for the Geostationary Satellites at 19 Degrees West". In: International Symposium on Space Dynamics. Toulouse, France, 1989.

[12] F. Fumenti et al. "Quasi-Impulsive Maneuvers to Correct Mean Orbital Elements in LEO". In: Proceedings of the 3rd CEAS EuroGNC. Toulouse, France, 2015.

[13] P. Gurfil et al. "The SAMSON Project - Cluster Flight and Geolocation with Three Autonomous Nano-satellites". In: 26th AIAA/USU Conference on Small Satellites. SSC12-VII-2. Salt Lake City, UT, USA, 2012.

[14] G. Hill. "Researches in the Lunar Theory". In: American Journal of Mathematics 1.1 (1878).

[15] D. LoBosco et al. "The Pleiades Fractionated Space System Architecture and the Future of National Security Space". In: AIAA Space Conference and Exposition. AIAA 2008-7687. San Diego, California: American Institute of Aeronautics and Astronautics, 2008.

[16] L. Mazal et al. "Closed-loop distance-keeping for long-term satellite cluster flight". In: Acta Astronautica 94.1 (2014).

[17] L. Mazal et al. "Cluster Flight Algorithms for Disaggregated Satellites". In: Journal of Guidance, Control, and Dynamics 36.1 (2013).

[18] D. Mishne. "Formation Control of Satellites Subject to Drag Variations and J2 Perturbations". In: Journal of Guidance, Control, and Dynamics 27.4 (2004).

[19] O. Montenbruck et al. "E/I-vector separation for safe switching of the GRACE formation". In: Aerospace Science and Technology 10.7 (2006).

[20] A. Moreira et al. "TanDEM-X: a TerraSAR-X add-on satellite for single-pass SAR interferometry". In: International Geoscience and Remote Sensing Symposium. Vol. 2. 2004.

[21] S. Persson et al. "PRISMA - Demonstration Mission for Advanced Rendezvous and Formation Flying Technologies and Sensors". In: 56th International Astronautical Congress. IAC-05B5.6.B.07. Fukuoka, Japan, 2005.

[22] C. W. T. Roscoe et al. "Formation Establishment and Reconfiguration Using Differential Elements in J2-Perturbed Orbits". In: Journal of Guidance, Control, and Dynamics 38.9 (2015).

[23] H. Schaub et al. "Hybrid Cartesian and Orbit Element Feedback Law for Formation Flying Spacecraft". In: Journal of Guidance, Control, and Dynamics 25.2 (2002).

[24] H. Schaub et al. "J $\mathrm{J}_{2}$ Invariant Relative Orbits for Spacecraft Formations". In: Celestial Mechanics and Dynamical Astronomy 79.2 (2001).

[25] H. Schaub et al. "Spacecraft formation flying control using mean orbit elements". In: Journal of the Astronautical Sciences 48.1 (2000).

[26] B. D. Tapley. "Gravity model determination from the GRACE mission". In: The Journal of the Astronautical Sciences 56.3 (2008).

[27] J. Tschauner et al. "Rendezvous zu Einem in Elliptischer Bahn um Laufenden Ziel". In: Acta Astronautica 11.5 (1965).

[28] D. R. Wood. "An algorithm for finding a maximum clique in a graph". In: Operations Research Letters 21.5 (1997).

[29] K. Yamanaka et al. "New State Transition Matrix for Relative Motion on an Arbitrary Elliptical Orbit". In: Journal of Guidance, Control, and Dynamics 25.1 (2002). 